\title{
6. VOLCANIC ROCKS AND TERTIARY CARBONATES DREDGED FROM THE CAGAYAN RIDGE AND THE SOUTHWEST SULU SEA, PHILIPPINES ${ }^{1}$
}

\author{
H. R. Kudrass, ${ }^{2}$ P. Müller, ${ }^{2}$ H. Kreuzer, ${ }^{2}$ and W. Weiss ${ }^{2}$
}

\section{INTRODUCTION}

The Cagayan Ridge divides the Sulu Sea into two basins. The $1500-2000 \mathrm{~m}$ deep northwest basin is underlain by a several kilometer thick layer of partly deformed sediments. In the 4000 $5000 \mathrm{~m}$ deep southeast basin the assumed oceanic basement is covered by sediments only $1 \mathrm{~km}$ thick (Murauchi et al., 1973; Mascle and Biscarrat, 1978; Rangin, 1989; Hinz et al., this volume). The northeastern part of the Cagayan Ridge faces the steep slope of the accretionary wedge of the subduction zone extending along the eastern rim of the southeast basin (Figs. 1 and 2). The northern section of the ridge is capped by numerous reef islands, where 400 -m-thick reef deposits were drilled at the Tubbataha Island. The central and southern part of the ridge is not as pronounced because some broad seamounts lie along the eastern side of the ridge.

Several hypotheses have been published explaining the origin of the Cagayan Ridge and the two basins of the Sulu Sea. According to Holloway (1981) the southeast basin of the Sulu Sea originated in back-arc spreading coincident in part with the opening of the South China Sea. In this model, the Cagayan Ridge formed in the Oligocene to mid-Miocene as a volcanic arc southeast of the accretionary wedge of Palawan.

Alternatively, the northward subduction of the Celebes Sea under the Sulu Archipelago is thought to have induced back-arc spreading forming the Sulu Sea (Hamilton, 1979; Mitchell et al., 1986; Rangin, 1989). The Cagayan Ridge originated in the Oligocene/early Miocene as a related volcanic arc along the southern margin of a fragment of the proto-China mainland. Lee and McCabe (1986) and others suggest entrapped Eocene oceanic basement as the origin of the southeast basin.

So far only one sample from the Cagayan Ridge was available, consisting of andesitic basalt with a K/Ar age of $14.7 \pm$ $0.6 \mathrm{Ma}$ (Kudrass et al., 1986). In this paper we describe dredge samples from the Cagayan Ridge and the southeast Sulu Basin obtained during two pre-site cruises with the Sonne $(1987,1988)$ (Table 1).

Dredge hauls were performed and sampling sites were determined as described by Kudrass (1986). Prospective sites were chosen along seismic records (Hinz et al., this volume) where basement outcrops were indicated, or on nearby steep slopes. Recovery ranges between some chips of rocks to $500 \mathrm{~kg}$. In one case, basement could be sampled by piston coring when two pebbles at the base of a slump deposit were recovered.

\section{VOLCANIC ROCKS}

Volcanic rocks were recovered from 12 sites on the Cagayan Ridge and from two seamounts in the southeast basin of the Sulu Sea. The rocks were analyzed by petrographic, geochemical, and $\mathrm{K}-\mathrm{Ar}$ methods.

\footnotetext{
${ }^{1}$ Rangin, C., Silver, E., von Breymann, M. T., et al., 1990. Proc. ODP, Init. Repts., 124: College Station, TX (Ocean Drilling Program).

2 Bundesanstalt für Geowissenschaften und Rohstoffe, Stilleweg 2, 3000 Hannover 51, Federal Republic of Germany.
}

According to their chemical composition the volcanic rocks can be classified as basalt, basaltic andesite, andesite, and dacite (Table 2, Fig. 3). Basalt was recovered from two seamounts in the southeast basin (Fig. 1): SO58-65 near ODP Site 769 and SO58-90 near Site 768. At the southern end of the Cagayan Ridge, near Maeander Reef, samples of basaltic andesite were dredged (SO23-15) or cored (SO49-96) as phosphatized pebbles from a slump deposit. Volcanic rocks from the northern end of the Cagayan Ridge (Fig. 2) are richer in silica and range from basaltic andesite to dacite (Fig. 3). Within this small sub-area, the composition varies independently of water depth or the position of the sample along the ridge.

In general, the basalts of the seamounts have a tholeitic composition, whereas the other volcanic rocks from the $\mathrm{Ca}$ gayan Ridge are predominantly calc-alkaline (Fig. 4).

Except for some volcanic sandstone and tuff, the volcanic rocks consist of mostly vesicular, phorphyritic rock commonly bearing phenocrysts of plagioclase, clinopyroxene, and some orthopyroxene (Table 3). Altered olivine occurs in the basalt of the seamounts and in the basaltic andesite from the southern end of the Cagayan Ridge. Phenocrysts of plagioclase up to 2 $\mathrm{cm}$ in length are present in each rock type, quite often including some glass aligned parallel to the zonal texture (Fig. 5). The plagioclase of the basaltic andesite of SO58-55-3 is especially rich in glassy inclusions that contain small bubbles of fluid and gas (Fig. 5).

The groundmass usually contains fine to microcrystalline laths of plagioclase and sometimes clinopyroxene. In some samples, light brown to light-colored glass is present. As a main component, glass occurs in the dacitic pumice (SO58-43-3) and is partly devitrified in smectitic sphaerolites (SO58-47-1).

The massive dacite (SO49-59) contains numerous large amphiboles of ferrian tschermakitic hornblende (after Leake, 1978 and Rock and Leake, 1984; Fig. 6; for chemical composition see Table 2), which are surrounded by a thin layer of opaque grains, except at the contact of the amphiboles with the labradorite phenocrysts (for chemical composition see Table 2). The unusual occurrence of this amphibole, which otherwise occurs in mesozonal metamorphic rocks, may be explained either by a resorption of an amphibolite by a dacitic lava or by a volcanogenic mobilization of amphibole-bearing metamorphic rocks. The divergent $\mathrm{K}-\mathrm{Ar}$ data of the amphibole (Table 4) also prove its xenolithic character.

\section{GEOCHEMISTRY}

The geochemical composition of the various volcanic rocks was used to characterize their volcano-tectonic origin.

As the samples have been obtained from outcrops, which were exposed for long periods to seawater, diagenetic alteration may have changed their composition. However, the petrographic analysis and the relative low concentration of LOI (mostly 2$4 \%$, Table 2) indicate that a severe alteration is limited to some samples only, which were excluded from the discussion. Another limitation is the X-ray fluorescence detection limit of about 5 to $15 \mathrm{ppm}$ for rare-earth elements and some other trace elements. 


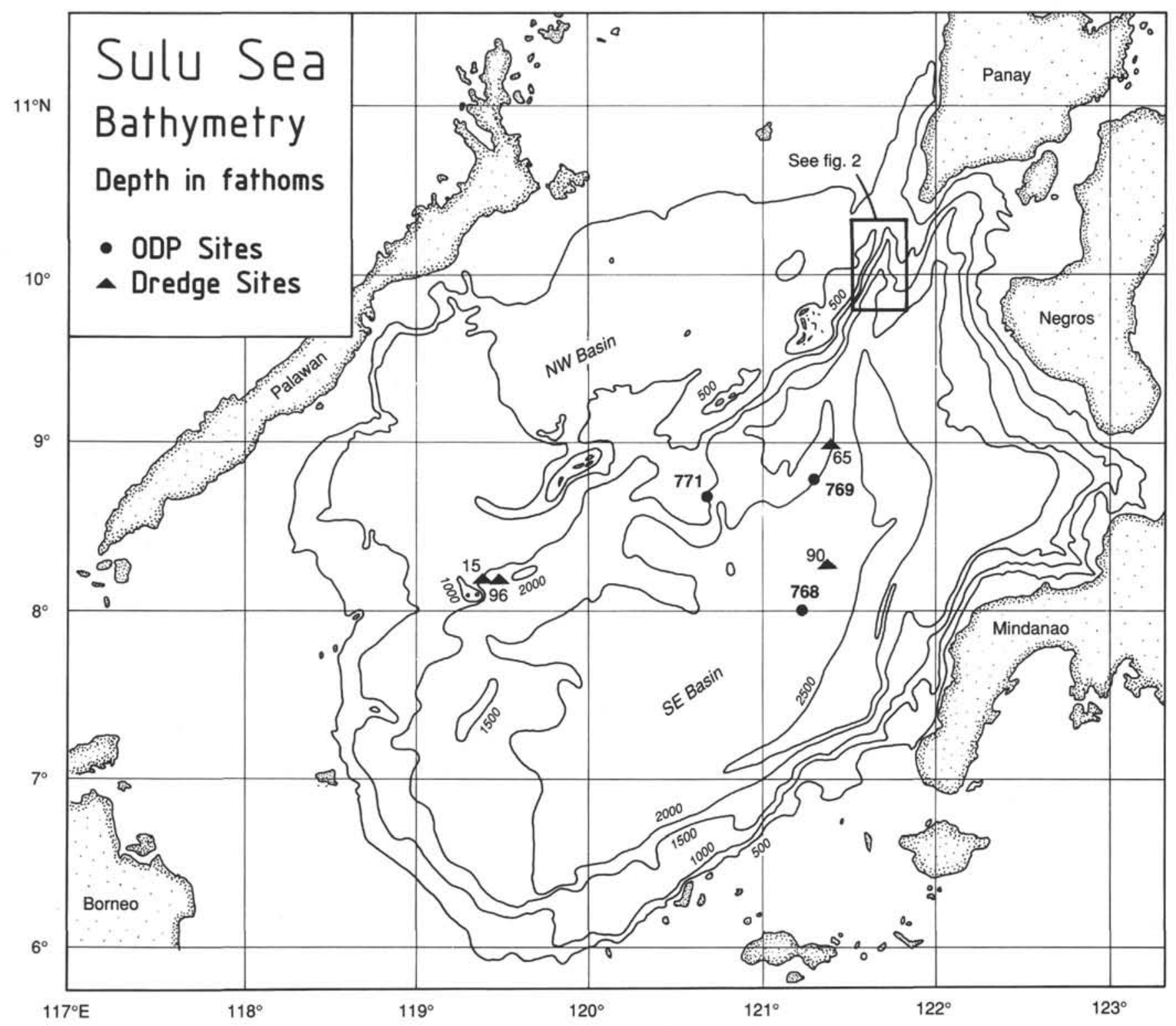

Figure 1. Location map of the Sulu Sea drill sites of ODP Leg 124.

The distribution of trace elements in the basalt samples from the two seamounts (SO58-65, -90, Fig. 7) is typical for transitional mid-ocean ridge basalt (E-MORB) after Pearce (1982). The great deviation of $\mathrm{P}$ in the samples of SO58-65 is presumably caused by a diagenetic phosphatization during the longterm exposure to seawater. The high variability of $\mathrm{P}_{2} \mathrm{O}_{5}$ from 0.7 to $2.6 \mathrm{wt} \%$, which correlates with an increase of $\mathrm{CaO}$ from 9.2 to $12.3 \mathrm{wt} \%$, indicates this alteration. Two pebbles from the Maeander Reef (SO49-96) were also phosphatized $\left(\mathrm{P}_{2} \mathrm{O}_{5}=6.06\right.$ wt \%). An 8-cm-thick manganese crust covering the outcrops of basalt of sample SO58-90 may have prevented this alteration $\left(\mathrm{P}_{2} \mathrm{O}_{5}\right.$-concentration 0.31 wt \%). The SO58-90 deficit of $\mathrm{Cr}$ with respect to E-MORB is presumably caused by early magmatic differentiation.

The intermediate and acid volcanic rocks from the Cagayan Ridge itself are difficult to interpret, as their original composition, indicative of their volcano-tectonic environment, is masked by fractional crystallisation and contamination. The basaltic andesite dredged from the Maeander Reef (SO23-15) fits to calcalkaline volcanic-arc basalts (Fig. 7); the rest of the higher dif- ferentiated rocks from the northern end of the Cagayan Ridge cannot be classified. Typically these volcanic rocks are extremely low in $\mathrm{Nb}$ and $\mathrm{Cr}$, usually below detection limits, poor in $\mathrm{TiO}_{2}$ (0.7-0.4 wt \%) and Ni (usually below $40 \mathrm{ppm}$ ).

\section{K-Ar DATA}

$\mathrm{K}-\mathrm{Ar}$ data were obtained from andesite (SO49-55) and dacite (SO49-59) samples dredged from the northern end of the Cagayan Ridge (Table 4, Fig. 2). In SO49-55 three varieties of andesite and in SO49-59 two varieties of dacite were investigated. Plagioclase (pl), amphibole (am), and rough-ground whole-rock (wr) were analyzed in different grain-size fractions, as well as one magnetic fraction ( $\mathrm{mf}$ ) of whole rock.

Usually, the relative standard deviations of the determinations of radiogenic argon and potassium are less than $1 \%$. But in the present case, the large scatter of the $\mathrm{K}-\mathrm{Ar}$ dates and the differences between the dates for different-size fractions of the same material suggest the presence of inhomogeneous material. Indeed, three duplicate analyses yielded differences in radiogenic argon of two to four times the possible analytical uncer- 


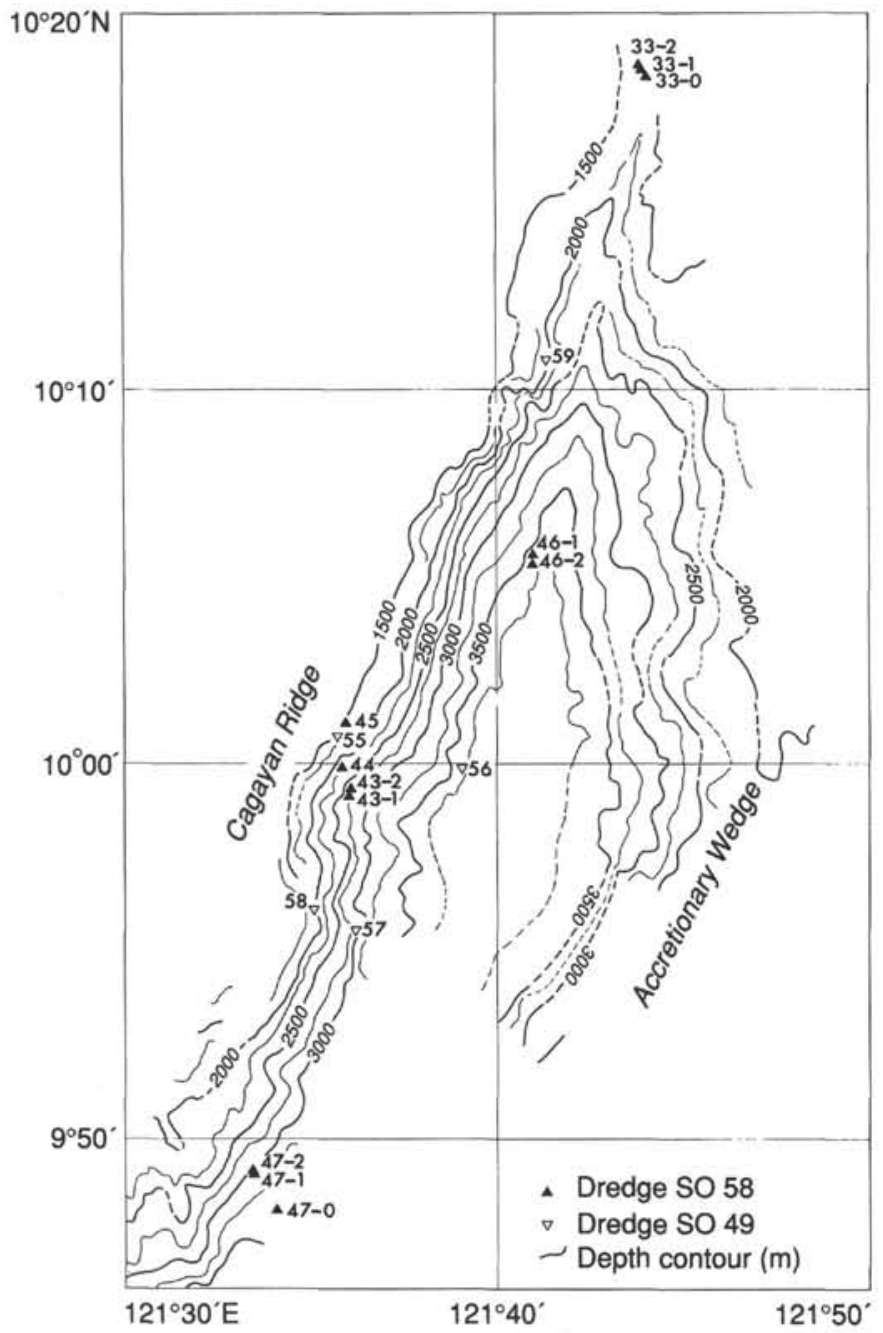

Figure 2. Detail map of the area outlined in Figure 1, Cagayan Ridge.
Table 1. Coordinates and water depths of dredge samples.

\begin{tabular}{|c|c|c|}
\hline Number & Coordinates (North/East) & $\begin{array}{l}\text { Water depth } \\
\text { (corrected) }\end{array}$ \\
\hline SO23-15 KD & $\begin{array}{r}8^{\circ} 10.390^{\prime} \\
119^{\circ} 21.800^{\prime}\end{array}$ & $3312 \mathrm{~m}$ \\
\hline SO49-55 KD & $\begin{aligned} 10^{\circ} 00.608^{\prime} \text { to } & 10^{\circ} 01.900^{\prime} \\
121^{\circ} 35.709^{\prime} & 121^{\circ} 35.890^{\prime}\end{aligned}$ & 2683 to $2014 \mathrm{~m}$ \\
\hline SO49-56 KD & $\begin{array}{r}9^{\circ} 59.860^{\prime} \\
121^{\circ} 39.065^{\prime}\end{array}$ & $3780 \mathrm{~m}$ \\
\hline SO49-57 KD & $\begin{array}{r}9^{\circ} 55.522^{\prime} \\
121^{\circ} 36.187^{\prime}\end{array}$ & $2677 \mathrm{~m}$ \\
\hline SO49-58 KD & $\begin{array}{r}9^{\circ} 56.076^{\prime} \text { to } 9^{\circ} 56.130^{\prime} \\
121^{\circ} 35.052^{\prime} \quad 121^{\circ} 34.700^{\prime}\end{array}$ & 2167 to $2065 \mathrm{~m}$ \\
\hline SO49-59 KD & $\begin{array}{r}10^{\circ} 10.791^{\prime} \\
121^{\circ} 41.339^{\prime}\end{array}$ & 2400 to $2100 \mathrm{~m}$ \\
\hline SO49-96 KL & $\begin{array}{r}8^{\circ} 11.10^{\prime} \\
119^{\circ} 28.05^{\prime}\end{array}$ & $3634 \mathrm{~m}$ \\
\hline SO58-33 KD & $\begin{array}{ll}10^{\circ} 18.26^{\prime} & 10^{\circ} 18.62^{\prime} \\
121^{\circ} 44.05 & 121^{\circ} 43.87\end{array}$ & 1650 to $1620 \mathrm{~m}$ \\
\hline SO58-43 KD & $\begin{array}{r}9^{\circ} 59.11^{\prime} \text { to } 9^{\circ} 59.33^{\prime} \\
121^{\circ} 35.97^{\prime} \quad 121^{\circ} 36.06^{\prime}\end{array}$ & 2700 to $2580 \mathrm{~m}$ \\
\hline SO58-44 KD & $\begin{array}{r}9^{\circ} 59.90^{\prime} \\
121^{\circ} 35.80^{\prime}\end{array}$ & $2220 \mathrm{~m}$ \\
\hline SO58-46 KD & $\begin{array}{r}10^{\circ} 05.59^{\prime} \text { to } 10^{\circ} 05.37^{\prime} \\
121^{\circ} 40.92^{\prime} \text { ' } 121^{\circ} 40.97^{\prime}\end{array}$ & 3580 to $3700 \mathrm{~m}$ \\
\hline SO58-47 KD & $\begin{array}{r}9^{\circ} 48.10^{\prime} \text { to } 9^{\circ} 49.20^{\prime} \\
121^{\circ} 34.02^{\prime}{ }^{\circ} 1^{\circ} 33.42^{\prime}\end{array}$ & 3220 to $2890 \mathrm{~m}$ \\
\hline SO58-65 KD & $\begin{array}{r}8^{\circ} 59.74^{\prime} \\
121^{\circ} 22.52^{\prime}\end{array}$ & $3500 \mathrm{~m}$ \\
\hline SO58-66 KD & $\begin{array}{r}9^{\circ} 00.30^{\prime} \\
121^{\circ} 23.45^{\prime}\end{array}$ & $3400 \mathrm{~m}$ \\
\hline SO58-90 KD & $\begin{array}{r}8^{\circ} 16.07^{\prime} \\
121^{\circ} 22.26^{\prime}\end{array}$ & $3600 \mathrm{~m}$ \\
\hline
\end{tabular}

tainty. We therefore calculate the error of the $\mathrm{K}$-Ar date with an uncertainty of the radiogenic argon arbitrarily enhanced by a factor of four. The only exceptions are the plagioclase dates for Samples SO49-55-3 and SO49-55-9, which have already large analytical uncertainties because of the relatively low concentrations of radiogenic argon.

The amiphibole (ferrian tschermakitic hornblende) in SO4955 exhibits the widest range in $\mathrm{K}-\mathrm{Ar}$ dates (36-158 Ma). Accord-

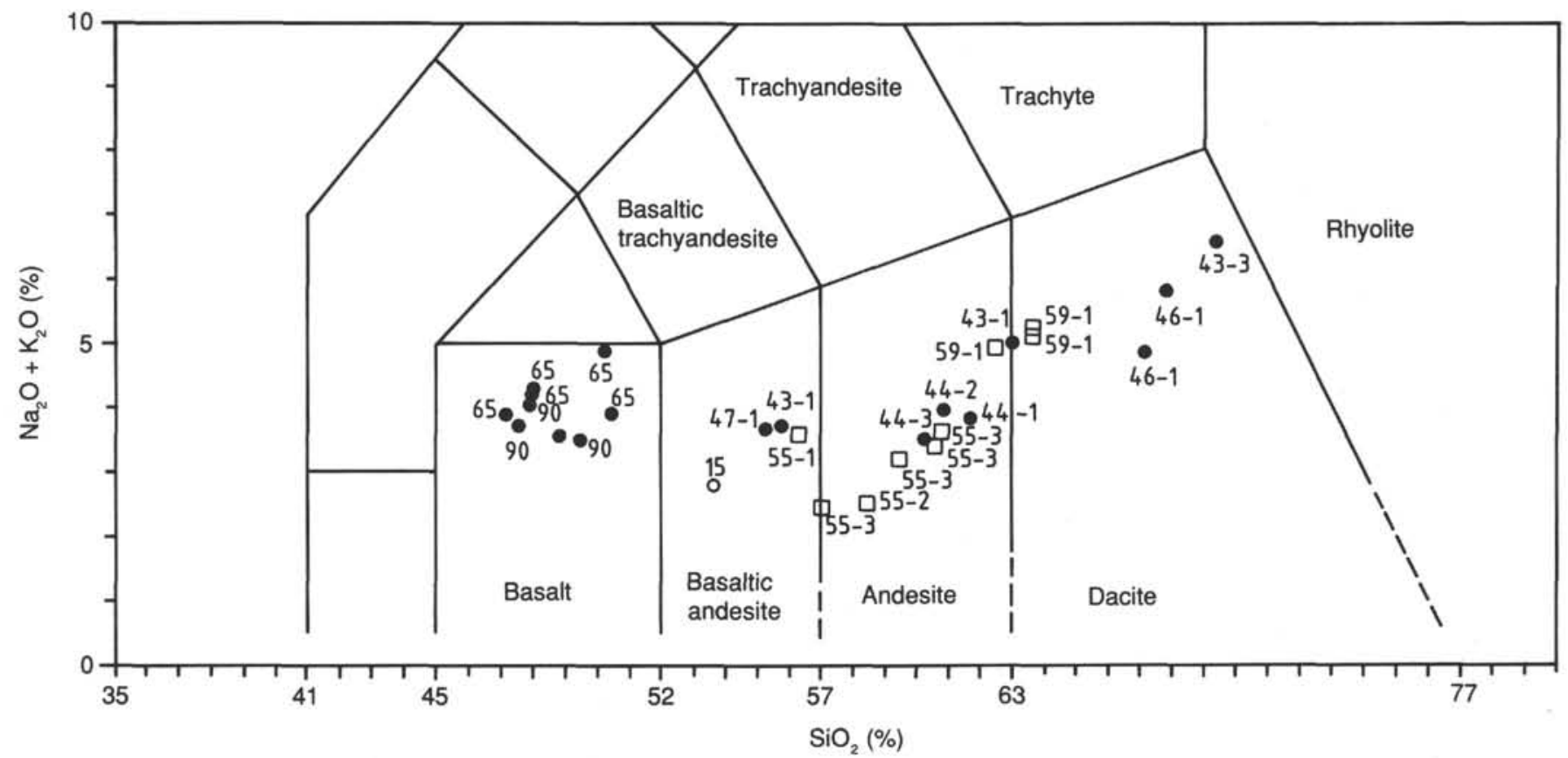

Figure 3. Chemical composition plot of volcanic rocks recovered from two seamounts in the southeast Sulu Basin. 
Table 2. Chemical composition of volcanites.

\begin{tabular}{|c|c|c|c|c|c|c|c|c|c|c|c|c|c|c|c|c|c|c|c|}
\hline & $\begin{array}{c}\text { SO23. } \\
15 \\
\text { wr }\end{array}$ & $\begin{array}{c}\text { SO } 49 . \\
55-1 \\
\text { wt }\end{array}$ & $\begin{array}{c}\text { SO49- } \\
55-2 \\
\text { lap }\end{array}$ & $\begin{array}{c}\text { SO49. } \\
\text { S5-3a } \\
\text { wr }\end{array}$ & $\begin{array}{c}\text { SO } 49 . \\
55-3 \mathrm{~b} \\
\text { wr }\end{array}$ & $\begin{array}{c}\text { SO49- } \\
55-3 \mathrm{c} \\
\text { wr }\end{array}$ & $\begin{array}{c}\text { SO49- } \\
\text { 5s-3d } \\
\text { wr }\end{array}$ & $\begin{array}{l}\text { SO49- } \\
57-2 a \\
\text { wr }\end{array}$ & $\begin{array}{l}\text { SO49- } \\
57-2 \mathrm{~b} \\
\mathrm{wr}\end{array}$ & $\begin{array}{l}\text { SO49- } \\
59.1 \mathrm{at} \\
\text { wr }\end{array}$ & $\begin{array}{c}\text { SO } 49 . \\
59-1 \mathrm{a} \\
\mathrm{am}\end{array}$ & $\begin{array}{c}\text { SO } 49 . \\
59-1 \mathrm{a} \\
\mathrm{pl}\end{array}$ & $\begin{array}{l}\text { SO49. } \\
59-1 \mathrm{a} \\
\mathrm{gm}\end{array}$ & $\begin{array}{l}\text { SO49- } \\
\text { 59-1b } \\
\text { wr }\end{array}$ & $\begin{array}{c}\text { SO49- } \\
59-1 \mathrm{~b} \\
\text { am }\end{array}$ & $\begin{array}{c}\text { SO } 49 .- \\
59-1 \mathrm{~b} \\
\mathrm{pl}\end{array}$ & $\begin{array}{c}\text { SO } 049- \\
59-1 \mathrm{~b} \\
\mathrm{gm}\end{array}$ & $\begin{array}{c}\text { SO } 049- \\
59-1 \mathrm{~b} \\
\text { wr }\end{array}$ & $\begin{array}{c}\text { SO49- } \\
96 \\
\text { wr }\end{array}$ \\
\hline SiO (wt. \%) & $\$ 2.13$ & 53.70 & 56.98 & 59.16 & 57.55 & 54.95 & 59.39 & 48.01 & 47.34 & 60.48 & 44.37 & 52.03 & 53.28 & 61.52 & 44.01 & 49.94 & 52.35 & 61.31 & 38.79 \\
\hline & 0.85 & 0.80 & 0.53 & 0.57 & 0.70 & 0.44 & 0.54 & 0.48 & 0.46 & 0.38 & 1.22 & 0.02 & 0.10 & 0.40 & 1.22 & 0.04 & 0.06 & 0.40 & 0.77 \\
\hline $\mathrm{Al}_{2} \mathrm{O}_{3}$ & 16.62 & 15.49 & 14.53 & 14.22 & 15.78 & 15.87 & 13.98 & 12.69 & 12.35 & 17.34 & 11.64 & 28.01 & 24.40 & 17.23 & 11.52 & 27.61 & 26.42 & 17.54 & 17.58 \\
\hline $\mathrm{Fe}_{2} \mathrm{O}_{3}$ & 9.62 & 10.60 & 8.72 & 7.53 & 7.98 & 8.05 & 7.49 & 9.94 & 9.50 & 5.54 & 15.29 & 0.49 & 1.65 & 5.59 & 15.34 & 0.71 & $\begin{array}{r}20.42 \\
1.10\end{array}$ & 4.85 & 5.59 \\
\hline $\mathrm{MnO}$ & 0.17 & 0.16 & 0.14 & 0.14 & 0.14 & 0.13 & 0.14 & 0.04 & 0.04 & 0.19 & 0.60 & 0.04 & 0.10 & 0.14 & 0.58 & 0.06 & 0.05 & 0.17 & 0.12 \\
\hline $\mathrm{MgO}$ & 5.09 & $\begin{array}{l}0.10 \\
3.60\end{array}$ & 5.47 & $\begin{array}{l}0.14 \\
4.37\end{array}$ & $\begin{array}{l}0.14 \\
4.33\end{array}$ & $\begin{array}{l}0.13 \\
5.81\end{array}$ & 5.11 & $\begin{array}{l}0.04 \\
8.74\end{array}$ & $\begin{array}{l}0.04 \\
8.18\end{array}$ & 2.15 & $\begin{array}{l}0.00 \\
12.11\end{array}$ & 0.10 & $\begin{array}{l}0.10 \\
1.21\end{array}$ & 2.20 & 12.49 & 0.98 & 1.17 & 2.36 & 2.23 \\
\hline $\mathrm{CaO}$ & 10.42 & 8.25 & 9.20 & 8.09 & 7.64 & 9.20 & 8.45 & 2.94 & 2.64 & 6.06 & 10.67 & 12.32 & 10.72 & 4.80 & 10.88 & 9.38 & 7.78 & 4.63 & 18.56 \\
\hline $\mathrm{Na}_{2} \mathrm{O}$ & 1.67 & 2,53 & 1.94 & 2.24 & 2.37 & 1.80 & 2.19 & 2.45 & 2.34 & 3.35 & 1.87 & 3.97 & 3.77 & 4.03 & 2.01 & 3.32 & 3.56 & 3.68 & 2.49 \\
\hline $\begin{array}{l}\mathrm{N}_{2} 2 \mathrm{O} \\
\mathrm{N}_{2} \mathrm{O}\end{array}$ & 1.04 & 0.89 & 0.51 & 1.29 & 0.73 & 0.56 & 1.13 & 1.54 & 1.72 & 1.41 & 0.39 & 0.55 & 0.89 & $\begin{array}{l}4.03 \\
0.90\end{array}$ & $\begin{array}{l}2.01 \\
0.39\end{array}$ & $\begin{array}{l}3.32 \\
1.17\end{array}$ & $\begin{array}{l}3.36 \\
1.24\end{array}$ & $\begin{array}{l}3.08 \\
1.35\end{array}$ & 0.86 \\
\hline $\mathrm{P}_{2} \mathrm{O}_{5}$ & 0.21 & 0.08 & 0.07 & 0.16 & 0.09 & 0.03 & 0.07 & 0.09 & 0.09 & 0.20 & 0.16 & 0.32 & 0.30 & 0.19 & 0.13 & 0.60 & 0.10 & 0.22 & 6.06 \\
\hline $\mathrm{SO}_{3}$ & 0.30 & 0.12 & 0.00 & 0.10 & 0.00 & 0.00 & 0.00 & 0.00 & 0.24 & 0.07 & 0.00 & 0,00 & 0.00 & 0.00 & 0.00 & 0.00 & 0.00 & .07 & 0.32 \\
\hline LOI & 1.38 & 3.13 & 1.56 & 1.50 & 2.44 & 2.55 & 1.18 & 12.80 & 14.75 & 2.44 & 1.19 & 1.89 & 3.04 & 2.58 & 1.01 & 5.58 & 5.75 & 3.04 & 6.10 \\
\hline Traces & 0.18 & 0.13 & 0.13 & 0.12 & 0.10 & 0.10 & 0.13 & 0.10 & 0.10 & 0.12 & 0.11 & 0.12 & 0.12 & 0.10 & 0.11 & 0.14 & 0.09 & 0.11 & 0.23 \\
\hline \multirow{2}{*}{\multicolumn{20}{|c|}{ Traces (ppm) }} \\
\hline & & & & & & & & & & & & & & & & & & & \\
\hline $\begin{array}{l}\text { As } \\
\mathrm{Ba}\end{array}$ & $\begin{array}{r}174 \\
174\end{array}$ & $\begin{array}{l}1 \\
79\end{array}$ & $\begin{array}{c}1 \\
55\end{array}$ & $\begin{array}{r}21 \\
108\end{array}$ & ${ }_{61}^{1}$ & $\begin{array}{l}1 \\
40\end{array}$ & $\begin{array}{l}7 \\
82\end{array}$ & 1 & $\begin{array}{l}14 \\
44\end{array}$ & $\begin{array}{r}234 \\
234\end{array}$ & 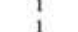 & $\begin{array}{l}1 \\
93\end{array}$ & $\begin{array}{l}10 \\
137\end{array}$ & $\begin{array}{r}1 \\
136\end{array}$ & 17 & $\begin{array}{l}19 \\
78\end{array}$ & $\begin{array}{l}1 \\
103\end{array}$ & 201 & $\begin{array}{r}9 \\
135\end{array}$ \\
\hline $\begin{array}{l}\mathrm{Ba} \\
\mathrm{Bi}\end{array}$ & $\begin{array}{r}1 / 4 \\
1\end{array}$ & 1 & 1 & 1 & $\begin{array}{r}01 \\
1\end{array}$ & $\begin{array}{r}40 \\
1\end{array}$ & $\begin{array}{r}82 \\
1\end{array}$ & i & $\begin{array}{r}44 \\
1\end{array}$ & $\begin{array}{r}234 \\
9\end{array}$ & $\begin{array}{l}1 \\
12\end{array}$ & $\begin{array}{r}93 \\
1\end{array}$ & $\begin{array}{r}137 \\
10\end{array}$ & $\begin{array}{r}136 \\
1\end{array}$ & $\begin{array}{l}1 \\
17\end{array}$ & $\begin{array}{r}78 \\
1\end{array}$ & $\begin{array}{r}103 \\
1\end{array}$ & $\begin{array}{r}201 \\
1\end{array}$ & $\begin{array}{r}135 \\
1\end{array}$ \\
\hline $\mathrm{Ce}$ & 47 & i & 1 & 1 & i & i & i & i & i & 1 & 21 & 1 & 1 & i & 20 & 1 & 1 & 1 & 130 \\
\hline Co & 12 & i & i & i & i & 21 & 24 & 31 & i & 1 & 21 & 1 & i & 1 & 23 & 1 & i & i & 1 \\
\hline $\mathrm{Cr}$ & 45 & i & 180 & 87 & 30 & 59 & 131 & 152 & 142 & 1 & 28 & 1 & 1 & 1 & 1 & 1 & i & i & 15 \\
\hline $\mathrm{Cu}$ & 83 & 97 & 40 & 63 & 25 & 49 & 71 & 41 & 48 & 13 & 19 & 11 & 15 & 14 & 21 & 120 & 7 & 13 & 77 \\
\hline $\mathrm{La}$ & 37 & 22 & & & 1 & 1 & 1 & 1 & 1 & 1 & 22 & 1 & 1 & 1 & 21 & 1 & 1 & 26 & 68 \\
\hline Mo & 1 & 5 & 5 & 5 & 4 & 1 & 1 & 1 & 1 & 5 & 1 & 1 & 1 & 1 & 4 & 1 & 1 & 5 & 1 \\
\hline $\mathrm{Nb}$ & 5 & 1 & 1 & 1 & 3 & 3 & 1 & i & i & 9 & 3 & 4 & 1 & 7 & 5 & 37 & 4 & 4 & 1 \\
\hline $\mathrm{Ni}$ & 30 & 10 & 74 & 26 & 19 & 26 & 30 & 54 & 46 & 8 & 14 & 1 & 1 & 5 & 12 & 1 & 1 & 1 & 33 \\
\hline $\mathrm{Pb}$ & 8 & 16 & 9 & 19 & 1 & 1 & 15 & 1 & 1 & 15 & 1 & 19 & 11 & 7 & 10 & 43 & 1 & 27 & 23 \\
\hline $\mathrm{Rb}$ & 46 & 26 & 12 & 27 & 13 & 15 & 23 & 22 & 33 & 36 & 7 & 11 & 21 & 15 & 8 & 17 & 19 & 15 & 14 \\
\hline Sc & 46 & 37 & 40 & 33 & 29 & 37 & 35 & 3 & 32 & 10 & 57 & 3 & 5 & 10 & 55 & 1 & 4 & & 30 \\
\hline Sn & 1 & 1 & 1 & 1 & 1 & 27 & 1 & 30 & 1 & 1 & 1 & 29 & 1 & 1 & 1 & 114 & 1 & 1 & 1 \\
\hline Sr & 388 & 149 & 138 & 173 & 160 & 143 & 155 & 89 & 89 & 366 & 87 & 820 & 642 & 307 & 78 & 599 & 561 & 306 & 711 \\
\hline $\mathrm{Ta}$ & 8 & 1 & 1 & 6 & 1 & I & 1 & 1 & 1 & 1 & 1 & 1 & I & 6 & 1 & 12 & 1 & 1 & i \\
\hline Th & 6 & 1 & 1 & 1 & 1 & 1 & 1 & 6 & 7 & 7 & 1 & 1 & 1 & 9 & 1 & 1 & 1 & 10 & 12 \\
\hline $\mathrm{u}$ & 1 & 1 & 1 & 1 & 1 & 1 & 1 & 6 & 1 & 1 & 1 & 1 & 1 & 1 & 5 & 1 & 1 & 1 & 42 \\
\hline v & 297 & 355 & 247 & 226 & 216 & 222 & 271 & 144 & 147 & 95 & 232 & 1 & 28 & 104 & 223 & 1 & 1 & 92 & 284 \\
\hline w & 1 & 22 & 1 & 6 & 1 & 1 & 1 & 1 & 11 & 1 & 1 & 1 & 1 & 1 & 1 & 1 & 1 & 1 & 24 \\
\hline $\mathrm{Y}$ & 19 & 26 & 26 & 20 & 24 & 19 & 21 & 12 & 16 & 1 & 6 & 6 & 11 & 1 & 68 & 9 & 9 & 15 & 103 \\
\hline $\mathrm{Zn}$ & 83 & 86 & 88 & 55 & 83 & 66 & 58 & 58 & 70 & 63 & 142 & 9 & 25 & 49 & 143 & 74 & 21 & 47 & 95 \\
\hline $\mathrm{Zr}$ & 45 & 46 & 56 & 70 & 66 & 51 & 74 & 20 & 26 & 95 & 53 & 23 & 42 & 93 & 58 & 22 & 33 & 88 & 40 \\
\hline
\end{tabular}

Notes: X-ray fluorescence analyses by J. Lodziak and D. Requard. Subsamples of one rock type are indicated by a, b, c...; LOI $=$ loss on ignition; wr $=$ whole rock; lap $=$ lapilli; gm $=$ groundmass; am $=$ amphibole; $\mathrm{pl}=$ plagioclase. Values of 1 indicate concentrations below detection limits.

ing to the petrographic analysis, this mineral is atypical for volcanites and may be derived as xenocrysts from a metamorphic rock. Apparently the transfer of the amphibole did not reset the K-Ar clock of the mineral, or the excess Ar from the metamorphic rock was not released. In any case, the old dates of the amphibole suggest the inheritance of argon from an older metamorphic rock.

The dates of the phenocrysts of plagioclase in SO49-59 have a smaller range ( 22 to $26 \mathrm{Ma}$ ), with the better-preserved plagioclase of SO49-59-7 yielding the younger dates. The dates for whole-rock fractions lie between the dates of the amphibole and the plagioclase in Sample SO49-59-1, but are comparable or even younger than those of the plagioclase in Sample SO49-59-7.

In the dilemma of proved excess argon and possible losses of argon from the plagioclase and even more from the whole-rock fractions, the youngest dates of SO49-59 (i.e., 22-26 Ma) provide only doubtful limits for the time of volcanic activity at the northernmost position of the Cagayan Ridge.

The K-Ar dates of SO49-55 are generally younger than those of SO $49-59$, but they also show a wide range (11 to $20 \mathrm{Ma}$ ) and a significant scatter for different fractions of the same sample. We have no certain criteria for the reliability of the different results. If the single date of the magnetic fraction is not considered, we have a cluster of two whole-rock samples, one plagioclase date around $15 \mathrm{Ma}$, and a single one at about $11 \mathrm{Ma}$ that might indicate the time of volcanic activity at the northern end of the Cagayan Ridge. The K-Ar date of $14.7 \pm 0.6 \mathrm{Ma}$ for a basaltic andesite (Kudrass et a1., 1986: SO23-15) suggests a similar time of volcanic activity at the southern end of the Cagayan Ridge. The deposition of the early to early middle Miocene shallow-water carbonates (see below) could be associated with this volcanic activity.

\section{SHALLOW-WATER CARBONATES}

At the northern end of the Cagayan Ridge, shallow-water carbonates were dredged at four localities with water depth ranging from 3800 to $1600 \mathrm{~m}$ (Fig. 2). The carbonates that consist of algal packstone, biomicrite or foraminifer sandstone contain shallow-water faunal assemblages of larger foraminifers (Lepidocyclina, Cycloclypeus, Table 5). The larger foraminifers that are frequently reworked indicate, at least for the Lepidocyclina species, an early Miocene to early middle Miocene age. The planktonic foraminifers from the younger sediments are of late Miocene (SO58-43) or of probable early Pliocene age (SO49-57).

The occurrence of early Miocene to early middle Miocene shallow-water carbonates, even when they are reworked into younger sediments, indicates that the Cagayan Ridge existed as a shallow area of presumably volcanic islands at that time. Early to middle Miocene carbonates were also dredged near the reef islands of the central Cagayan Ridge (Kudrass, 1987).

\section{SUMMARY}

The tholeiitic basalt samples dredged from two seamounts near ODP Sites 768 and 769 have a trace-element distribution comparable to transitional mid-ocean ridge basalts (after Pearce, 1982). The volcanic rocks obtained from the Cagayan Ridge on which Site 769 was drilled are richer in silica and range from basaltic andesite to dacite. Most of the rocks belong to the calc-alkaline series, possibly indicating that the Cagayan Ridge 
Table 2 (continued).

\begin{tabular}{|c|c|c|c|c|c|c|c|c|c|c|c|c|c|c|c|c|c|c|c|c|}
\hline $\begin{array}{c}\text { SO58. } \\
43-1 \mathrm{a} \\
\text { lap }\end{array}$ & $\begin{array}{c}\text { SO58- } \\
43-1 \mathrm{~b} \\
\text { lap }\end{array}$ & $\begin{array}{c}\text { SO58. } \\
43-3 \\
\text { wr }\end{array}$ & $\begin{array}{c}\text { SO58- } \\
43-6 \\
\text { wr }\end{array}$ & $\begin{array}{c}\text { SO58. } \\
44-1 \mathrm{a} \\
\text { wr }\end{array}$ & $\begin{array}{c}\text { SO58- } \\
44-1 b \\
\text { wr }\end{array}$ & $\begin{array}{c}\text { SO58. } \\
44-1 \mathrm{c} \\
\text { wr }\end{array}$ & $\begin{array}{c}\text { SO58- } \\
46-1 \mathrm{a} \\
\text { wr }\end{array}$ & $\begin{array}{c}\text { SOS8- } \\
46-1 \mathrm{~b} \\
\text { wr }\end{array}$ & $\begin{array}{c}\text { SO58- } \\
46-5 \\
\text { wr }\end{array}$ & $\begin{array}{c}\text { SO58- } \\
47-1 \\
\text { lap }\end{array}$ & $\begin{array}{c}\text { SO58- } \\
47-3 \\
\text { wr }\end{array}$ & $\begin{array}{c}\text { SOS8- } \\
65-1 \mathrm{a} \\
\text { wr }\end{array}$ & $\begin{array}{c}\text { SOS8- } \\
65-1 \mathrm{~b} \\
\text { wr }\end{array}$ & $\begin{array}{c}\text { SO58- } \\
65-1 \mathrm{c} \\
\text { wr }\end{array}$ & $\begin{array}{c}\text { SO58- } \\
65-1 d \\
\text { wr }\end{array}$ & $\begin{array}{c}\text { SO58- } \\
65-2 \\
\text { wr }\end{array}$ & $\begin{array}{c}\text { SOS8- } \\
90-1 \mathrm{a} \\
\text { wr }\end{array}$ & $\begin{array}{c}\text { SOS8- } \\
90-1 b \\
\text { wr }\end{array}$ & $\begin{array}{c}\text { SO58- } \\
90-2 \\
\text { wr }\end{array}$ & $\begin{array}{c}\text { SOs8- } \\
90-3 \\
\text { wr }\end{array}$ \\
\hline 52.53 & 56.53 & 63.67 & 22.71 & 60.37 & 59.23 & 59.39 & 65.31 & 66.41 & 50.54 & 47.76 & 43.83 & 43.95 & 45.85 & 43.96 & 46.23 & 43.17 & 46.13 & 47.16 & 45.14 & 44.81 \\
\hline 0.67 & 0.73 & 0.53 & 0.27 & 0.47 & 0.46 & 0.46 & 0.65 & 0.62 & 0.72 & 0.78 & 1.98 & 1.18 & 1.12 & 1.15 & 1.08 & 1.08 & 1.22 & 1.26 & 1.41 & 1.38 \\
\hline 16.02 & 14.21 & 13.07 & 3.46 & 14.30 & 14.25 & 14.30 & 15.32 & 15.48 & 13.49 & 15.83 & 16.72 & 18.37 & 17.85 & 18.53 & 17.06 & 16.74 & 19.06 & 20.23 & 19.76 & 19.44 \\
\hline 9.59 & 7.37 & 3.78 & 6.24 & 6.83 & 7.29 & 6.96 & 4.93 & 4.15 & 7.95 & 9.03 & 14.40 & 9.30 & 9.12 & 9.35 & 9.99 & 9.37 & 10.01 & 9.19 & 10.40 & 10.38 \\
\hline 0.11 & 0.16 & 0.15 & 1.28 & 0.14 & 0.14 & 0.15 & 0.11 & 0.07 & 0.20 & 0.12 & 0.21 & 0.09 & 0.08 & 0.12 & 0.62 & 0.51 & 0.22 & 0.14 & 0.14 & 0.18 \\
\hline 5.73 & 2.45 & 1.19 & 9.67 & 4.42 & 5.22 & 4.64 & 1.26 & 0.86 & 4.17 & 4.94 & 7.75 & 2.78 & 3.48 & 2.74 & 3.01 & 2.77 & 5.06 & 4.30 & 3.87 & 4.16 \\
\hline 6.62 & 4.00 & 3.34 & 27.45 & 7.91 & 8.61 & 8.15 & 4.97 & 4.62 & 8.18 & 5.30 & 6.03 & 11.29 & 9.20 & 11.01 & 9.70 & 12.34 & 9.79 & 10.01 & 9.96 & 10.69 \\
\hline 3.12 & 3.15 & 4.46 & 0.98 & 2.46 & 2.43 & 2.65 & 3.92 & 4.54 & 3.14 & 2.23 & 4.36 & 2.85 & 2.99 & 3.05 & 2.60 & 2.72 & 2.84 & 2.88 & 3.16 & 2.98 \\
\hline 0.39 & 1.34 & 1.57 & 0.32 & 1.29 & 1.02 & 1.21 & 0.80 & 1.15 & 3.73 & 0.94 & 0.07 & 1.00 & 1.47 & 0.88 & 0.99 & 0.85 & 0.54 & 0.46 & 0.64 & 0.53 \\
\hline 0.10 & 0.20 & 0.12 & 0.26 & 0.06 & 0.07 & 0.07 & 0.17 & 0.14 & 0.38 & 0.08 & 0.15 & 1.34 & 0.70 & 1.34 & 1.07 & 2.58 & 0.29 & 0.31 & 0.34 & 0.32 \\
\hline 0.00 & 0.00 & 0.05 & 0.36 & 0.00 & 0.00 & 0.00 & 0.00 & 0.00 & 0.45 & 0.00 & 0.00 & 0.00 & 0.00 & 0.00 & 0.00 & 0.03 & 0.00 & 0.00 & 0.00 & 0.00 \\
\hline 4.38 & 9.50 & 7.76 & 26.40 & 1.21 & 0.99 & 1.75 & 2.25 & 1.59 & 6.63 & 12.63 & 3.91 & 7.30 & 7.68 & 7.21 & 6.64 & 7.34 & 4.51 & 3.20 & 4.68 & 4.79 \\
\hline 0.12 & 0.09 & 0.07 & 0.27 & 0.12 & 0.13 & 0.12 & 0.08 & 0.08 & 0.29 & 0.09 & 0.13 & 0.18 & 0.18 & 0.20 & 0.20 & 0.19 & 0.16 & 0.15 & 0.15 & 0.16 \\
\hline 99.37 & 99.72 & 99.75 & 99.68 & 99.58 & 99.84 & 99.84 & 99.77 & 99.71 & 99.87 & 99.73 & 99.53 & 99.65 & 99.71 & 99.54 & 99.18 & 99.70 & 99.82 & 99.28 & 99.66 & 99.82 \\
\hline 1 & 1 & 1 & 1 & 1 & 1 & 1 & 14 & 15 & 1 & 1 & 1 & 1 & 1 & 22 & 1 & 22 & 1 & 1 & 1 & 1 \\
\hline 48 & 94 & 96 & 32 & 104 & 101 & 111 & 84 & 91 & 295 & i & i & 66 & 84 & 70 & 169 & 147 & 82 & 70 & 74 & 54 \\
\hline 1 & 1 & 1 & 17 & 1 & 1 & 1 & 1 & 1 & 1 & i & 11 & 1 & 1 & 1 & 1 & 1 & 1 & 1 & 1 & 1 \\
\hline i & 1 & 1 & 1 & 1 & $i$ & i & i & i & i & i & $i$ & i & i & 1 & 23 & i & 1 & 1 & i & i \\
\hline 42 & 1 & 1 & 61 & 34 & 24 & 31 & i & i & 54 & 25 & 74 & 1 & 1 & 1 & 39 & 23 & 25 & i & 1 & i \\
\hline 1 & i & i & 794 & 125 & 157 & 98 & $i$ & $i$ & 491 & 1 & 47 & 300 & 339 & 383 & 213 & 226 & 63 & 15 & i & 21 \\
\hline 58 & 35 & 27 & 58 & 55 & 59 & 70 & 14 & 25 & 77 & 60 & 10 & 96 & 70 & 89 & 110 & 103 & 61 & 52 & 59 & 135 \\
\hline 1 & 1 & 1 & 1 & 1 & 1 & 1 & 1 & 1 & 1 & 1 & 1 & 29 & 1 & 1 & 23 & 1 & 1 & 1 & 1 & 1 \\
\hline 1 & 4 & 5 & 1 & 6 & 6 & 3 & $i$ & i & $i$ & $i$ & $i$ & 5 & i & i & 4 & i & i & i & 4 & 4 \\
\hline i & 4 & 4 & i & 6 & 1 & 1 & $i$ & $i$ & $i$ & 5 & 4 & 17 & 11 & 12 & 13 & 13 & 13 & 12 & 13 & 14 \\
\hline 10 & 1 & 1 & 587 & 25 & 26 & 28 & i & 1 & 139 & 1 & 37 & 60 & 69 & 65 & 94 & 85 & 54 & 19 & 26 & 36 \\
\hline 1 & i & i & 7 & 1 & 11 & 17 & 7 & 13 & 1 & 1 & 1 & 1 & 1 & 1 & 14 & 1 & 1 & 9 & 11 & 1 \\
\hline 13 & 21 & 26 & 9 & 30 & 19 & 26 & 14 & 25 & 39 & 15 & 4 & 15 & 24 & 15 & 22 & 14 & 7 & 11 & 9 & 8 \\
\hline 45 & 24 & 14 & 13 & 29 & 38 & 29 & 19 & 19 & 29 & 29 & 52 & 37 & 39 & 35 & 35 & 33 & 30 & 32 & 32 & 35 \\
\hline 1 & 1 & 1 & 1 & 1 & 1 & 1 & 1 & 1 & 1 & 1 & 1 & 1 & 1 & 1 & 1 & 1 & 1 & 23 & 1 & 1 \\
\hline 167 & 194 & 153 & 195 & 170 & 153 & 179 & 193 & 185 & 724 & 180 & 114 & 272 & 248 & 280 & 269 & 302 & 338 & 354 & 349 & 359 \\
\hline 1 & 1 & 1 & I & 1 & 1 & 1 & 1 & 1 & i & 1 & 1 & 1 & 1 & 1 & 1 & 1 & 1 & 10 & 1 & 1 \\
\hline 1 & i & i & i & i & i & i & i & i & $i$ & 1 & $i$ & $i$ & $i$ & i & 9 & i & 1 & 1 & I & 6 \\
\hline i & i & i & $i$ & 6 & $i$ & $i$ & $i$ & i & $i$ & 1 & 1 & 1 & 7 & 1 & 1 & 1 & 7 & 6 & $i$ & 1 \\
\hline 365 & 101 & 27 & 117 & 189 & 210 & 200 & 70 & 80 & 237 & 187 & 397 & 248 & 207 & 260 & 225 & 225 & 300 & 311 & 325 & 317 \\
\hline 1 & i & 1 & 1 & 1 & 1 & 1 & 1 & 1 & 1 & 1 & 1 & 1 & 1 & 1 & 1 & 1 & 1 & $i$ & 1 & 1 \\
\hline 23 & 36 & 30 & 10 & 25 & 20 & 24 & 32 & 33 & 14 & 14 & 39 & 34 & 37 & 26 & 57 & 33 & 19 & 24 & 24 & 22 \\
\hline 69 & 89 & 64 & 41 & 50 & 57 & 48 & 70 & 53 & 75 & 80 & 63 & 120 & 108 & 116 & 113 & 116 & 103 & 100 & 108 & 115 \\
\hline 32 & 77 & 86 & 19 & 75 & 57 & 66 & 86 & 89 & 53 & 46 & 85 & 80 & 75 & 77 & 74 & 70 & 95 & 74 & 86 & 90 \\
\hline
\end{tabular}

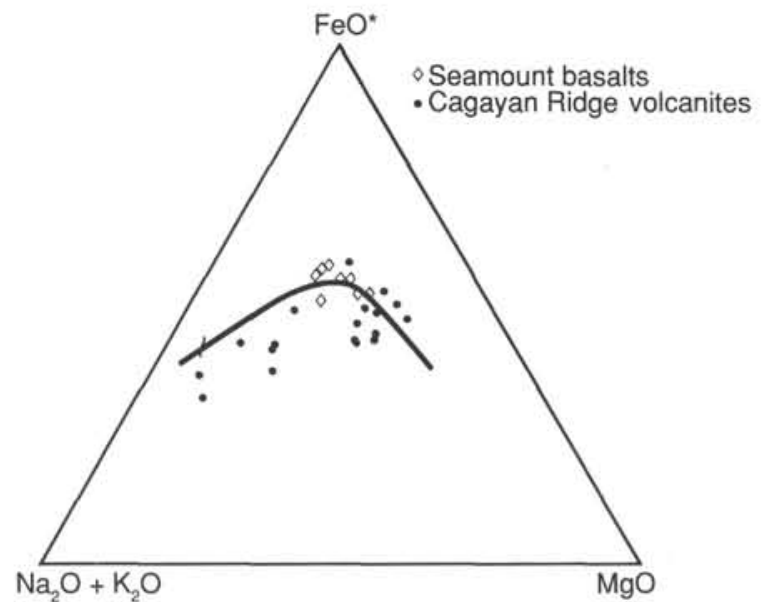

Figure 4. Classification of volcanic rocks from the two southeast Sulu Basin seamounts and the Cagayan Ridge. Separating line between tholeiitic (upper part) and calk-alkaline basalts is after Irvine and Baragar (1971).

originated as a volcanic arc. The $\mathrm{K}-\mathrm{Ar}$ dates of basaltic rocks and sites from the northern end of the Cagayan Ridge are inconsistent but suggest that the volcanic activity occurred in a period between 10 to $20 \mathrm{Ma}$. At approximately the same time, shallow-water carbonates of early to early middle Miocene age were deposited on the slopes of presumably volcanic seamounts or islands in the central and northern Cagayan Ridge. Dacites from the northernmost Cagayan Ridge (SO48-59) bear xeno- crysts of possible metamorphic origin, which obviously contain excess argon.

\section{REFERENCES}

Bideau, D., and Hekinian, R., 1984. Segregation vesicles of ocean floor basalts, 1. Petrological study of the segregation products. J. Geophys. Res., 89:7903-7914.

Hamilton, H., 1979. Tectonics of the Indonesian region. (U.S.) Geol. Surv. Prof. Pap., No. 1078.

Holloway, N. H., 1981. The North Palawan Block, Philippines: its relation to the Asian mainland and its role in the evolution of the South China Sea. Bull. Geol. Soc. Malays., 14:19-58.

Irvine, T. N. and Baragar, H.R.A, 1971. A guide to the chemical classification of the common volcanic rocks. Can. J. Earth Sci., 8:523547.

Kudrass, H. R., 1987. Improvement of deep-sea dredging operations through the use of a depth meter. J. Sediment. Petrol., 54:39-43.

Kudrass, H. R., Hiedicke, M., Cepek, P., Kreuzer, H., and Müller, P., 1986. Mesozoic and Cenozoic rocks dredged from the South China Sea (Reed Bank area) and Sulu Sea and their significance for platetectonic reconstructions. Mar. Pet. Geol., 3:19-30.

Leake, B. E., 1978. Nomenclature of amphiboles. Can. Mineral., 16: 501-520.

Lee, C. S., and McCabe, R., 1986. The Banda Celebes Sulu Basin: a trapped piece of Cretaceous-Eocene oceanic crust? Nature, 322: 51-54.

Le Bas, M. J., Le Maitre, R. H., Streckeisen, A., and Zanettin, B., 1986. A chemical classification of volcanic rocks based on the total alkali-silica diagram. J. Petrol., 27:745-750.

Mascle, A., and Biscarrat, P. A., 1978. The Sulu Sea: a marginal basin in Southeast Asia. In Watkins, J. S., et al. (Eds.), Geophysical Investigations of the Continental Margins. AAPG Mem., 29:373-381.

Mitchell, A.H.G., Hernandez, F., and Dela Cruz, A. P., 1986. Cenozoic evolution of the Philippines Archipelago. J. Southeast Asia Earth Sci., 1:3-22. 
Murauchi, S., Ludwig, H. J., Den, N., Hotta, H., Asanuma, T., Yoshi, T., Kuboten, A., and Agiwara, K., 1973. Structure of the Sulu Sea and Celebes Sea. J. Geophys. Res., 78:3437-3447.

Pearce, J. A., 1982. Trace-element characteristic lavas from destructive plate boundaries. In Thorpe, R. S. (Ed.), Orogenic Andesites and Related Rocks: New York (Wiley-Interscience), 525-548.

Rangin, C., 1989. The Sulu Sea, a back-arc basin setting within a Neogene collision zone. Tectonophysics, 161:119-141.

Rock, N.M.S., and Leake, B. E., 1984. The International Mineralogical Association amphibole nomenclature scheme: computerization and its consequences. Mineral. Mag., 48:211-227.

Seidel, E., Kreuzer, H., and Harre, H., 1982. A late Oligocene/early Miocene high pressure belt in the external Hellenides. Geol. Jahrb., Ser $E: 165-206$

Steiger, R., and Jäger, E., 1977. Subcommission on Geochronology: convention on the use of decay constants in geo- and cosmochronology. Earth Planet. Sci. Lett., 36:359-362.

Ms 124A-106

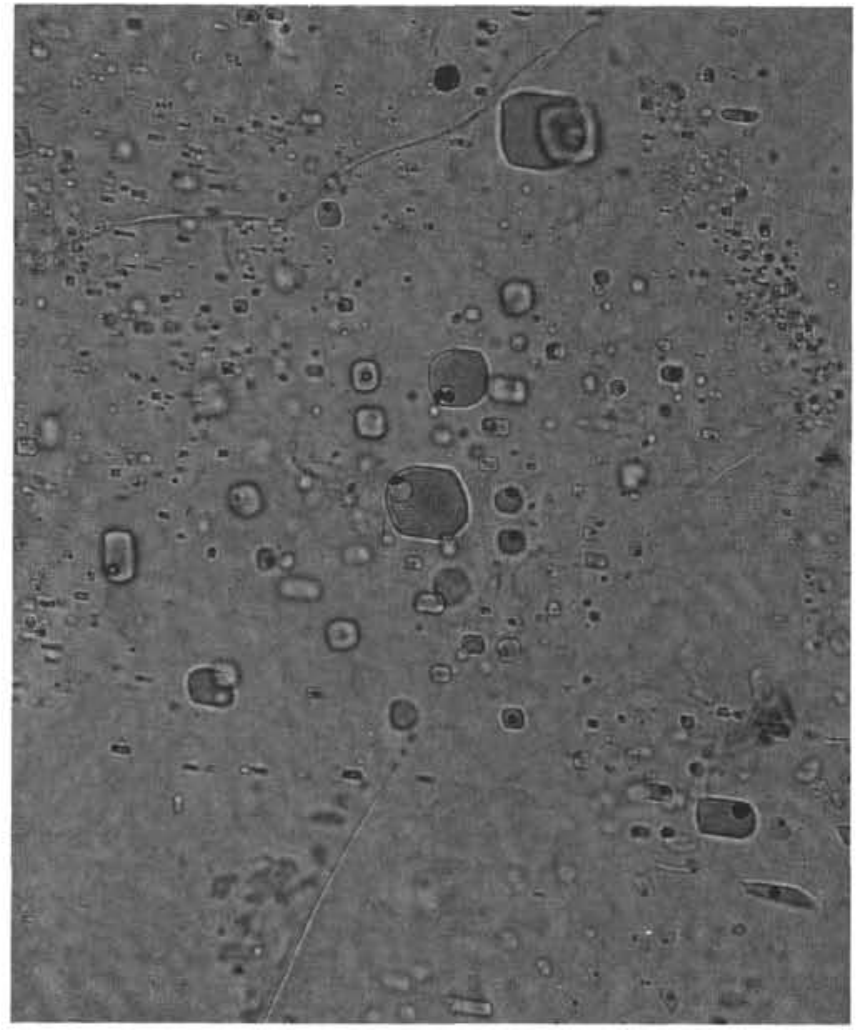

Figure 5. Photograph of phenocrysts of plagioclase with glass inclusions in basalts and basaltic andesite from southeast Sulu Basin seamounts and the Cagayan Ridge. Sample SO49-55-3.
Table 3. Description of the mineralogical composition of volcanites.

\section{Sample \\ Number}

Description

SO23-15 Porphyritic basaltic andesite, some vesicles.

Pc: pl, cpx, some altered ol.

$\mathrm{Gm}$ : microcrystalline, pl, pyroxene in light-brown glass.

SO49-55-1 Vesicular porphyritic andesite, fine and large vesicles.

Pc: pl with inclusions of glass and small cpx.

$\mathrm{Gm}$ : brown glass with laths of $\mathrm{pl}$ and $\mathrm{cpx}$, fluidal texture.

SO49-55-2 Lapilli tuff, lapilli consisting of fine-vesicular porphyritic andesite.

Pc: pl with inclusions of glass; some cpx; cpx with inclusions of glass and op. $\mathrm{Gm}$ : fine crystalline with $\mathrm{pl}, \mathrm{cpx}$, op.

SO49-55-3 Vesicular porphyritic andesite.

Pc: zonal pl with inclusions of glass, fluid, and gas; cpx; some opx (hypersthene) with inclusions of glass.

$\mathrm{Gm}$ : glass with some $\mathrm{pl}$ and $\mathrm{cpx}$, devitrifiaction sphaerolites.

Glass in pe darker than in gm.

SO49-55-9 Fine-vesicular porphyritic andesite.

Pc: zonal pl with inclusions of glass, cpx, and op; opx; cpx; agglomerates of $\mathrm{pl}, \mathrm{cpx}$, and opx.

Gm: fine crystalline with needles of $\mathrm{pl}$, op; smectite in vesicles.

SO49-57-2 Tuff with lapilli of basaltic andesite, lapilli with fluidal texture.

SO49-59-1 Massive porphyritic dacite.

Pc: green am (ferrian-tschermakitic hornblende) and pl (labradorite), both pc contain glassy inclusions, rim of op surrounds am, however not when am contacts $\mathrm{pl}$, large $\mathrm{pl}$ have often smecitic or carbonate cores, other $\mathrm{pc}$ are apatite, op.

$\mathrm{Gm}$ : microcrystalline with ?quartz, pl, op, and am.

SO49-59-7 Massive porphyritic dacite similar to SO49-59-1, but somewhat better preserved (no carbonate in $\mathrm{pl} \mathrm{pc).} \mathrm{Gm}$ with chlorite aggregates partly around phlogopite, am pc are not always surrounded by op, some $\mathrm{cm}$-sized microdioritelike restites.

SO49-96 Two pebbles of highly vesicular porphyritic andesite.

Pc: pl, some cpx, altered (?) ol.

$\mathrm{Gm}$ : microcrystalline laths of $\mathrm{pl}$, grains of $\mathrm{cpx}$, op, brown glass.

The rim of the pebbles is phosphatized.

SO58-33-4 Reddish or grey tuffite.

SO58-43-1 Basaltic or andesite grey ash with lapilli consisting of highly fine-vesicular andesite or basaltic andesite.

Pc: some $\mathrm{pl}$ and $\mathrm{cpx}$.

$\mathrm{Gm}$ : small laths of pl exhibit fluidal texture.

SO58-43-3 White to light gray ash with dacitic pumice, pumice with faint fluidal texture. Pc: pl, cpx, op.

Gm: light colored glass.

SO58-43-6 Greenish carbonaceous, smectitic, sandy tuffite (pl, cpx, green am, brown am, quartz).

Rare grains of calcareous algae.

SO58-44 Fine-vesicular andesite.

Pc: $\mathrm{pl}$ (up to $2 \mathrm{~cm}$ in diameter), opx, $\mathrm{cpx}$, op, large $\mathrm{pl}$ are corroded and contain many inclusions of glass; medium-sized pl contain some glass inclusions.

Gm: brown glass, pl needles, op grains.

SO58-46-1 Fine-vesicular porphyritic dacite.

Pc: pl, opx, some cpx, op; pc often in aggregates.

Pm: brown glass, very fine crystalline with small needles of $\mathrm{pl}$, some $\mathrm{cpx}$, fluidal texture.

SO58-46-5 Zeolithic, smectitic volcanic sandstone consisting of pl, light-green $\mathrm{cpx}$, green and brown am, biotite, and rare planktonic foraminifers, calcareous algae.

SO58-47-1 Ash with various lapilli, light-brown lapilli consist of andesite to dacitic devitrified glass, vesicles filled with smectite; dark-brown lapilli consist of devitrified glass, vesicles filled with smectite; dark-brown lapilli
basaltic andesite with small laths of pl in cryptocrystalline gm.

SO58-47-3 Greenish slightly porphyritic metamorphite (prasinite-like) with chemical composition of basaltic andesite, intersertal texture.

Pc: laths of pl, altered cpx.

Gm: fine crystalline, pl, chlorite, epidote, leucoxene, op, am, vesicles filled with epidote, chlorite, one pebble only, probably an artifact.

SO58-65 Slightly porphyritic basalt with large and small round vesicles (segregation vesicles corresponding to those described by Bideau and Hekinian (1984)).

Pc: altered ol, some pl.

Gm: cryptocrystalline, intersertal texture, many laths of $\mathrm{pl}$, some cpx, op. Vesicles partly lined by zeolites.

Outcrop covered by a 8 -cm-thick manganese crust.

SO58-90-1 Slightly porphyritic basalt with large vesicles and microporosity.

Pc: pl, cpx, altered ol, some opx.

$\mathrm{Gm}$ : fine crystalline intersertal texture, smectitic glass with laths of pl, cpx, op, some skeletal opx.

Large vesicles lined by zeolite, small vesicles partly filled by smectite.

SO58-90-2 As SO58-90-1, but in $\mathrm{gm}$ am (kaersutite) in radial aggregates. 


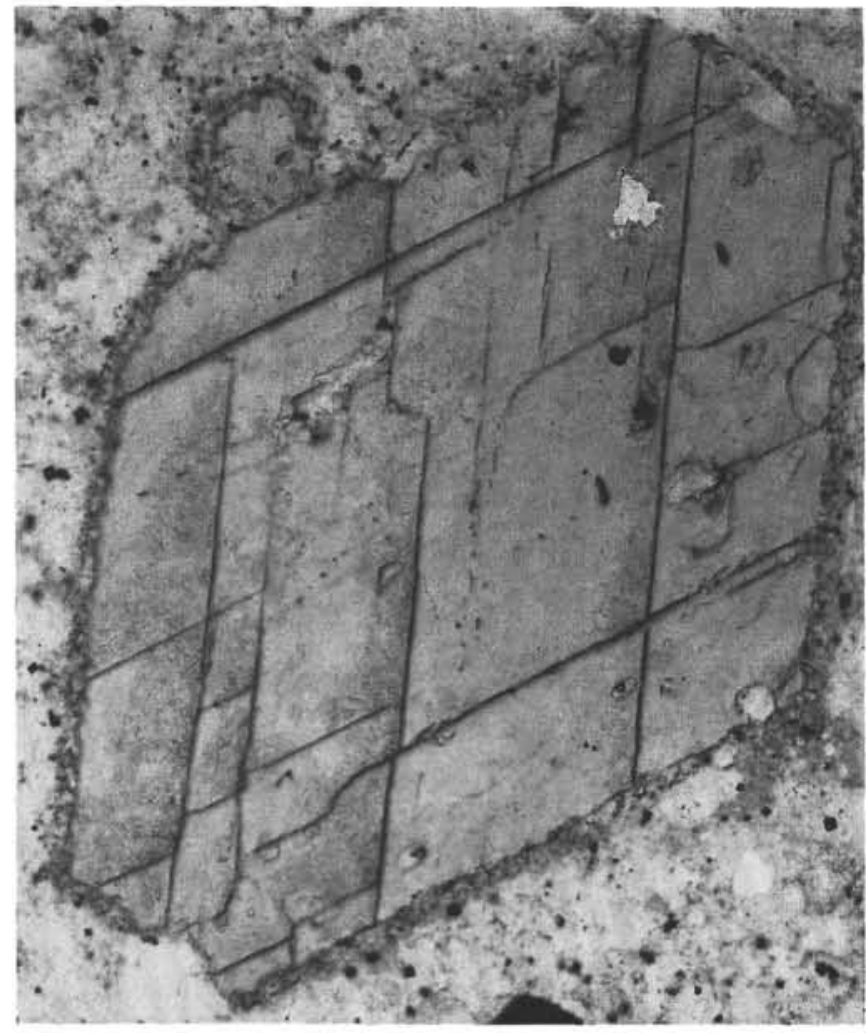

Figure 6. Photograph of dacitic Sample SO49 showing large amphiboles of ferrian tschermakitic hornblende. Sample SO49-59-1.
Table 4. K-Ar data. Argon determined by standard total fusion massspectrometric isotope-dilution analysis and potassium by flame photometry.e.g., (Seidel et al., 1982).

\begin{tabular}{|c|c|c|c|c|c|}
\hline $\begin{array}{l}\text { Sample no. } \\
\text { Rock type }\end{array}$ & $\begin{array}{l}\text { Material } \\
\text { size }(\mu \mathrm{m})\end{array}$ & $\begin{array}{l}\text { K-Ar date } \\
\text { (Ma) }\end{array}$ & $\underset{\text { (wt \%) }}{K}$ & $\begin{array}{l}\mathrm{Ar}_{\mathrm{rad}} \\
(\mathrm{nl} / \mathrm{g})\end{array}$ & $\begin{array}{l}\mathrm{Ar}_{\text {atm }} \\
\text { (STP) }\end{array}$ \\
\hline SO $49-55-1$ & wr $(200-100)$ & $14.9 \pm 0.5$ & 0.476 & 0.28 & 1.28 \\
\hline $\begin{array}{l}\text { Porphyritic } \\
\text { andesite }\end{array}$ & wr $(100-63)$ & $16.6 \pm 0.9$ & 0.442 & 0.29 & 1.09 \\
\hline SO49-55-3 & mf $(200-100)$ & $19.8 \pm 0.7$ & 1.181 & 0.914 & 0.24 \\
\hline $\begin{array}{l}\text { Basaltic } \\
\text { andesite }\end{array}$ & $\mathrm{pl}(100-63)$ & $14.7 \pm 1.1$ & 0.115 & 0.066 & 0.38 \\
\hline $\begin{array}{l}\text { SO49-55-9 } \\
\text { Porphyritic } \\
\text { andesite }\end{array}$ & pl (100-63) & $10.7 \pm 1.5$ & 0.147 & 0.061 & 0.32 \\
\hline SO49-59-1 & wr $(600-400)$ & $36.5 \pm 0.7$ & 0.930 & 1.33 & 0.74 \\
\hline \multirow{5}{*}{$\begin{array}{c}\text { Porphyritic } \\
\text { dacite }\end{array}$} & wr $(400-250)$ & $50.5 \pm 1.6$ & 0.913 & 1.82 & 0.77 \\
\hline & am $(250-160)$ & $158 \pm 5$ & 0.347 & 2.23 & 0.23 \\
\hline & am $(160-80)$ & $36.5 \pm 1.3$ & 0.335 & 0.48 & 0.30 \\
\hline & pl $(250-160)$ & $25.2 \pm 1.3$ & 0.979 & 0.98 & 2.93 \\
\hline & $\mathrm{pl}(160-80)$ & $25.8 \pm 1.1$ & 0.978 & 0.98 & 2.88 \\
\hline SO49-59-7 & wr $(630-315)$ & $21.8 \pm 0.7$ & 1.50 & 1.28 & 0.47 \\
\hline \multirow{5}{*}{$\begin{array}{c}\text { Porphyritic } \\
\text { dacite }\end{array}$} & wr $(315-200)$ & $22.0 \pm 0.7$ & 1.46 & 1.26 & 0.41 \\
\hline & am $(200-125)$ & $66.1 \pm 2.3$ & 0.331 & 0.87 & 0.34 \\
\hline & am (125-63) & $60.5 \pm 2.1$ & 0.323 & 0.77 & 0.34 \\
\hline & pl (200-125) & $22.3 \pm 0.9$ & 0.464 & 0.40 & 0.55 \\
\hline & $\mathrm{pl}(125-63)$ & $23.9 \pm 1.0$ & 0.446 & 0.42 & 0.52 \\
\hline $\begin{array}{l}\text { SO23-15 } \\
\text { Porphyritic } \\
\text { basaltic } \\
\text { andesite }\end{array}$ & $\begin{array}{l}\text { wr } \\
\text { (Kudrass et al., 1986) }\end{array}$ & $14.7 \pm 0.6$ & 0.863 & 0.49 & 0.18 \\
\hline
\end{tabular}

Notes: IUGS recommended constants used (Steiger and Jäger, 1977). Argon in nannoliters per gram at standard pressure and temperature. Error estimates at a level of $95 \%$ confidence in the analytical value (see text). Abbreviations: wr = sieve fraction of crushed whole-rock, $\mathrm{mf}=$ magnetic fraction of $\mathrm{wr}, \mathrm{am}=$ amphibole, $\mathrm{pl}=$ plagioclase. 


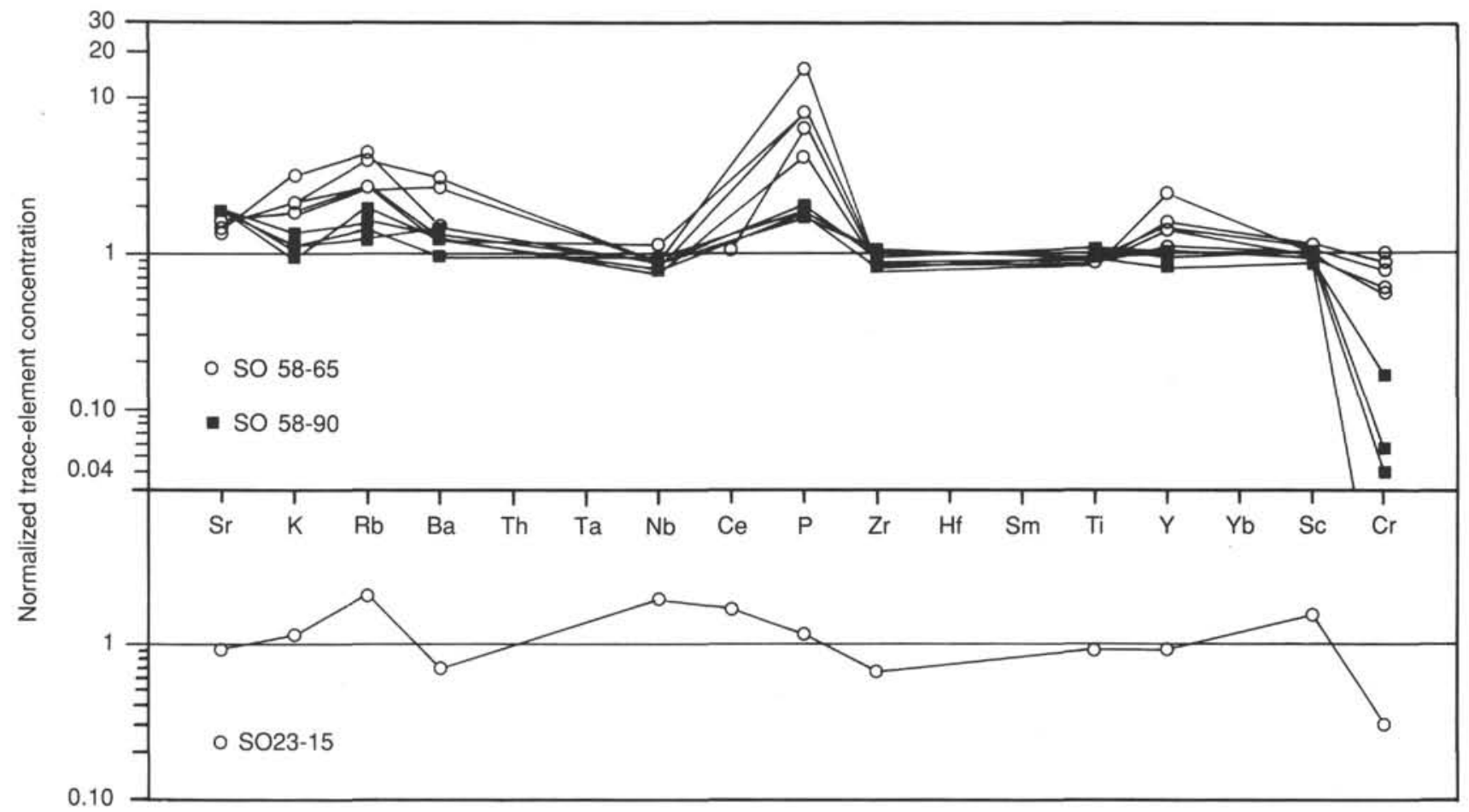

Figure 7. Trace elements in basalts. Upper part: The two seamounts of the southeast Sulu Basin, normalized to E-MORB basalt after Pearce (1982). Lower part: Andesitic basalt from the southwestern Cagayan Ridge, normalized to volcanic arc calc-alkaline basalt after Pearce (1982).

Table 5. Microfacies, foraminifer fauna, and age of shallow-water carbonates from the northern edge of the Cagayan Ridge.

\begin{tabular}{|c|c|c|}
\hline $\begin{array}{c}\text { Sample } \\
\text { no. }\end{array}$ & Microfacies and foraminifer fauna & Age and remarks \\
\hline SO $49-56$ & $\begin{array}{l}\text { Algal packstone with larger foraminifers reworked, } \\
\text { such as Lepidocyclina (Nephrolepidina) aff. ferreri } \\
\text { Provale, Cycloclypeus sp., Operculina sp., Amphis- } \\
\text { tegina } \mathrm{sp} \text {. }\end{array}$ & $\begin{array}{l}\text { early Miocene to early } \\
\text { middle Miocene }\end{array}$ \\
\hline SO $49-57$ & $\begin{array}{l}\text { Algal-foraminifer wacke-/packstone with reworked } \\
\text { Lepidocyclina specimens, and with Amphistegina } \\
\text { sp., Heterostegina } \mathrm{sp} ., \text { Gypsina sp., Calcarina sp., } \\
\text { Globorotalia tumida (Brady), Sphaeroidinella } \\
\text { specimens }\end{array}$ & $\begin{array}{l}\text { Probably early Pliocene with } \\
\text { reworked early to middle } \\
\text { Miocene }\end{array}$ \\
\hline SO $49-58$ & $\begin{array}{l}\text { Biomicrite with algal fragments and larger forams, } \\
\text { such as } L \text {. aff. verbeeki (Newton and Holland), } \\
\text { Cycloclypeus sp. and Amphistegina sp. and with } \\
\text { planktonic foraminifers, such as Globigerinoides } \\
\text { sp.sp. and Orbulina universa D'orbigny }\end{array}$ & early middle Miocene \\
\hline SO $58-43$ & $\begin{array}{l}\text { Slightly cemented planktonic foraminifer sand with rare } \\
\text { benthic and larger foraminifers; with Globoquad- } \\
\text { rina altispira (Cushman and Jarviz), Globoquad- } \\
\text { rina dehiscens (Campman, Parr and Collins), } \\
\text { Globorotalia aff. merotumida Blow and Banner, } \\
\text { Globorotalia aff. plesiotumida Blow and Banner, } \\
\text { Orbulina suturalis Brönnimann and others, and } \\
\text { with reworked specimens of Cycloclypeus sp., } \\
\text { Lepidocyclina sp., and Planorbulina } \mathrm{sp} .\end{array}$ & $\begin{array}{l}\text { Probably late Miocene with } \\
\text { reworked early to middle } \\
\text { Miocene }\end{array}$ \\
\hline
\end{tabular}

